\title{
Special Collection: Celebrating J.D. Murray's Contributions to Mathematical Biology
}

\author{
Philip K. Maini ${ }^{1}$ - Mark A. J. Chaplain ${ }^{2}$. Mark A. Lewis ${ }^{3}$. \\ Jonathan A. Sherratt ${ }^{4}$ \\ Published online: 4 December 2021 \\ (c) The Author(s), under exclusive licence to Society for Mathematical Biology 2021
}

In the mid-1960s, after a distinguished early career in fluid dynamics, Jim Murray realised how important it was for mathematicians to become involved in biology, where the term is used in its broadest sense to also include medicine, epidemiology and ecology. Thus, he became one of the founders of modern mathematical biology, tackling an astonishingly diverse range of subjects with vision, originality and creativity. Here, we select a few examples that illustrate his great versatility.

In 1983, Murray proposed a new model for self-organisation in biological pattern formation. The prevailing model at the time was the coupled system of reactiondiffusion equations proposed by Turing who hypothesized that spatial pattern arose due to cells responding to chemical pre-patterns, set up by the phenomenon of diffusiondriven instability (Turing 1952). It was known that mesenchymal cells generate large contractile forces that deform the extracellular environment. This observation formed the new model proposed by Murray and colleagues (Oster et al. 1983; Murray et al. 1983) in which it was hypothesized that these forces led to cells aggregating into self-organised patterns. This was a completely new way of looking at the problem of self-organisation in tissues and they showed how the resultant system of highly nonlinear partial differential equations led to patterns that were consistent with those observed in two well-studied examples, namely, skin-organ primordia (specifically feather germs) and skeletal patterning in the vertebrate limb. Murray was one of the very first people to combine mechanics with biochemistry, developing what is now known as the mechanochemical theory of pattern formation. This theory found

$凶 \quad$ Philip K. Maini

philip.maini@maths.ox.ac.uk

1 Wolfson Centre for Mathematical Biology, Mathematical Institute, University of Oxford, Oxford OX2 6GG, UK

2 School of Mathematics and Statistics, Mathematical Institute, University of St Andrews, St Andrews KY16 9SS, UK

3 Department of Mathematical and Statistical Sciences, CAB 545B, University of Alberta, Edmonton, AB T6G 2G1, Canada

4 Department of Mathematics, Heriot-Watt University, Edinburgh EH14 4AS, UK 
application in a range of areas, including the propagation of post-fertilization waves on eggs (Lane et al. 1987).

In parallel with this new approach to pattern formation, Murray extended the classical pre-pattern reaction-diffusion theory to study the effects of domain size and growth. A particularly striking application of this theory is to animal coat markings (Murray 1981, 1988). He further developed this approach to address the issue of how patterns evolved in the context of evolution (Oster et al. 1988). Using a chemotaxis model, he showed that the sex-dependent stripe patterns exhibited by the alligator Alligator mississippiensis could be explained by incubation temperature, and did not necessarily have to be genetically sex-linked (Murray et al. 1990). A similar modelling framework was proposed to account for the diverse pigmentation patterning observed on snakes (Murray and Myerscough 1991).

Both mechanochemical and reaction-diffusion mechanisms were then combined to study the initiation and sequential positioning of teeth in Alligator mississippiensis (Sneyd et al. 1993). This model also accounts for jaw growth, age structure of epithelial cells, and age-dependent production of cell adhesion molecules, and shows that these added complexities are necessary to account for the observed patterning.

Murray also explored cell movement in a different context, namely that of epidermal wound healing, in a series of papers that proposed that the biochemical regulation of mitosis is key to the healing of wounds (Sherratt and Murray 1990). The model was then used to make predictions on the effects on wound closure of regulating mitosis and wound shape (Sherratt and Murray 1992). While the aforementioned papers addressed adult epithelial wound healing, Murray also investigated epithelial wound healing in embryos, where the mechanism is quite different, involving the contraction of actin cables at the wound edge (Sherratt et al. 1992). Further work involved deriving a mechanical model for dermal wound healing which allowed for a study of how mechanical effects on, and remodelling of, extracellular matrix could affect the extent of scar tissue formation (Tracqui et al. 1995a, b).

Murray proposed a number of models in epidemiology to investigate disease spread. For example, Murray et al. (1986) uses a simple model for the transmission of rabies among foxes to quantify its spread should the disease be introduced to England. Parameter values were determined from the literature (and calculated from data) and the model used to investigate the effectiveness of different control strategies (for example, vaccination versus culling). Bentil and Murray (1993) used age-structured and non-age-structured models to investigate the spread of bovine tuberculosis infection in badgers. The model was analysed using a logical parameter search method to determine the values the model parameters must take to exhibit key types of observed behaviours. The values are then shown to be in good agreement with those from the literature. Nelson et al. (2000) presents a model for HIV AIDS which includes a delay in virus production when a cell is infected, as well as accounting for the fact that drugs are not $100 \%$ efficent. A detailed analysis of this model improves upon previous estimates of infected cell loss rates.

In ecology, the papers of Lewis and Murray (1993) and White et al. (1996a,b) developed and analysed novel models for wolf pack territory formation that took into account movement, scent marking, and predator-prey interactions. Analyses of these models revealed, amongst other results, steady states that contained buffer zones 
devoid of large numbers of wolves. Not only did this agree with field observations, it also suggested a mechanism for how prey, such as deer and moose, may survive in areas next to their main predator. This work has implications more generally and, in particular, for the social organization of humans. Indeed, in Volume II of his book, Mathematical Biology, Murray extends these ideas in the context of intertribal warfare (Murry 2003).

In the mid-1990s Murray began work on modelling the growth of brain tumours (glioma) in a series of highly influential papers upon which, some 25 years on, much ongoing research is still building. Tracqui et al. (1995a, b) considered a simple model of glioma cell proliferation and infiltration. Fitting model simulations to computerized tomography (CT) scans using optimization techniques allowed the model to be parametrised and then used to predict the effect of chemotherapy on the spread of the tumour, accounting for sensitive and resistant cell populations. Woodward et al. (1996) used a similar model to explore different surgical resection strategies. While these papers investigated the model on two-dimensional cross-sections of the brain, Burgess et al. (1997) extended the modelling to three spatial dimensions. An important model prediction here is that cell diffusion was actually a more important component of glioma growth than proliferation rate.

A further significant extension involved taking into account for the first time, to our knowledge, spatial heterogeneity within the brain. Specifically, Swanson et al. (2000) extended the model to incorporate the enhanced rate of cell motility in white matter as compared to that in grey matter. This paper used magnetic resonance imaging (MRI) and CT imaging data to simulate tumour growth on an anatomically accurate brain domain. The inclusion of grey and white matter then allowed the model to predict pathways of probable tumour infiltration, thus identifying areas of the brain that may be more susceptible to invasion and should therefore be targeted for treatment. Moreover, the model was used to predict how much of an expanding tumour could be missed due to the limitations of image detection methods and how this then allows us to begin to determine the true extent of invasion, which has important surgical implications. The importance of using mathematical models as virtual tumours to complement and enhance information gained from imaging and inform therapy is highlighted in Swanson et al. (2002) and the review article Swanson et al. (2003).

In the social sciences, as well as addressing intertribal conflict (as mentioned above), Murray has also proposed a mathematical model for the dynamics of marital interaction. Couples were observed and their interactions described via a Rapid Couples Interaction Scoring System (RCISS). A mathematical model, consisting of a coupled system of nonlinear discrete (in time) equations, was then developed to account for how various interactions contributed to each partner's RCISS score (Cook et al. 1995). Analysis of the model revealed a number of steady states, each of which describes a certain type of marriage. By parametrising the model from data on a particular couple's interactions, the model was found to be able to predict the probability of divorce, in a fixed period of time, to a very high degree of accuracy. The model was also used to predict how changing specific aspects of a couple's behaviour would affect their relationship (Gottman et al. 2002, Murray 2003).

The above is just a small sample of Murray's ground-breaking research and, while his personal research has been hugely influential on the development of mathematical 
biology, it is only one part of his contribution. Right from the start of his work on biological applications, he collaborated with experimental biologists. In doing so, he helped to establish a yardstick for genuinely applied mathematics in the life sciences that now permeates the thinking and policy-making in the field. Murray has also been extremely successful in attracting people from other areas of applied mathematics into mathematical biology. His infectious enthusiasm for the subject is an important part of this, coupled with the wide range of exciting biological problems he has at hand, and his incredible ability to get to the core of the problem, seek out the key question, and find a way to answer it.

In 1983 Murray established the Oxford Centre for Mathematical Biology, through which he organised a wide-ranging visitor programme. A high proportion of today's senior figures in mathematical biology spent time at the Centre in the 1980s, and were strongly influenced by its interdisciplinary ethos and collaborative spirit. Furthermore, he has trained and mentored a generation of mathematical biologists as graduate students and postdocs, inspiring creativity and originality in their work. Many of these trainees now hold influential positions in academic departments around the world.

Murray's book Mathematical Biology, first published in 1989 and then updated to the present version comprising two volumes (Murry 2002, 2003), is one of the most influential books published in the field. It forms the essential text for most high level mathematical biology courses taught worldwide and has been translated into several different languages. It is one of the reasons why mathematical biology has been transformed from a niche subject to an established part of most university mathematics courses.

Murray has received many deserved accolades during his career. He is a Fellow of the Royal Society, a Fellow of the Royal Society of Edinburgh, and a Foreign Member of the French Academy. He was awarded the London Mathematical Society (LMS) Naylor Prize (1988-1990), the Society of Mathematical Biology (SMB) Akira Okubo prize (2005), the Royal Society Bakerian Medal and Prize Lecture (2009), the Institute of Mathematics and its Applications (IMA) Gold Medal (2009), the European Academy of Sciences Leonardo da Vinci Medal (2011) and the William Benter Prize in Applied Mathematics (2012). He has also been awarded honorary doctorates by several universities, and, in 2006, the University of Washington created the James D, Murray Chair of Neuropathology, a donor endowed chair in perpetuity.

This Special Collection celebrates the diversity of Jim Murray's contribution to the field of mathematical biology. It contains 17 invited articles and, here, we briefly outline what is in each article.

While Murray's work investigated certain areas of tumour growth and therapy, mathematical modelling is now being used to address many different aspects of cancer treatment. Cassidy et al. (2020) addresses the problem of the toxic side-effects of cytotoxic chemotherapy treatment for cancer. Building on previous work that proposed strategies to reduce chemotherapy-induced neutropenia (lack of circulating neutrophils), this paper extends the model to include monocyte production. Using clinical data to determine model parameters, it is shown that monocytopenia precedes neutropenia and hence that monocytropenia can be used as a clinical marker to facilitate the delivery of an optimal dosing strategy to reduce, or completely eliminate, neutropenia. 
Murray's ground-breaking work on glioma has been extended in many different directions as advances in experimental technology reveal new observations. Curtin et al. (2020) extends a previous model of glioblastoma (GBM) to investigate clinical observations that suggest that distal tumour recurrence was more likely to occur in cases of ischemia following surgery. A nutrient-transport equation is added to the original model, and is parametrized using glucose uptake rates in GBM. The model is then used to determine which mechanisms are most likely to result in distal recurrence and it is found that tumours with faster migration and slower proliferation rates are more likely to recur in response to ischemia. A detailed simulation study is carried out to investigate different recurrence scenarios.

Murray's work on spatial disease spread and how it can be controlled, most famously on rabies in foxes, has inspired many researchers to ask similar questions for different disease systems. Anita et al. (2021) ask how bacterial disease spreads spatially in olive trees via insects and how this can be controlled via management strategies such as weed cut, treated nets and resistant cultivars. They find that a "containment band", where control measures are judiciously applied, is sufficient to prevent overall spread of the disease. This is in some ways similar to the earlier discovery by Murray and colleagues that a vaccination band ahead of the rabies front could be sufficient to contain its spread.

Murray's work on pattern formation highlighted how important domain geometry is for pattern selection. While his work in this area was primarily at the tissue level, Seirin-Lee (2021) shows that geometry may also play a role at the cell level. This paper develops a partial differential equation model for cytoplasmic polarity within a cell in the context of asymmetric cell division. The model describes the interaction of key membrane proteins with those in the bulk of the cell. A detailed numerical simulation study illustrates how cell geometry can affect the observed behaviour of the model and shows that to understand how cell polarity is established in asymmetric cell division, geometry must be taken into account.

Kulesa et al. (2021) highlights how Murray's approach to mathematical modelling "offers a practical template for constructing clear, logical, direct and verifiable models that help to explain complex cell behaviors and direct new experiments" by presenting a brief review of how this approach, used by the authors, has led to new insights into neural crest cell biology and cancer. The paper shows how mathematical models have been used to test and generate hypotheses on the mechanisms that underly collective cell movement in neural crest. In particular, it illustrates how a cell-based mechanistic model identified a number of phenotypic traits that must be possessed by cells, and how these were then validated using gene sequencing and bioinformatics. Furthermore, it is shown how understanding normal development, in the context of neural crest cell migration, can help us understand key aspects of cancer cell metastasis.

While Murray's work on HIV AIDS investigated aspects of within-host dynamics, the paper by Levy et al. (2021) focuses on population spread and investigates the effect of stigma. A mechanistic model for stigma is proposed and incorporated into a model for infection dynamics. The models are parameterised by fitting to data from Kenya and then the full model is used to make predictions on how different components of stigma affect the spread of the disease. The study highlights the importance of 
gathering data on sociological processes when investigating the spread of infectious diseases.

Murray has worked extensively on the biochemical regulation of cell behaviour in a wide range of physiological contexts. Sneyd et al. (2021) reviews work of this type for the specific case of saliva secretion by acinar cells in the salivary glands. The paper paints a picture of a continual interplay between experiments and modelling, highlighting the effectiveness of models in modifying and enhancing our understanding of saliva generation. The paper concludes with a discussion of the new generation of models currently being developed to utilise the most recent empirical data.

Woolley et al. (2021) addresses the issue of complex spatial pattern formation by building not only on Murray's work but, literally, on Murray himself (see Figure 10 and Figure 11). This paper shows how a two-morphogen reaction-diffusion Turing model can be built so that the parameter space in which patterning occurs, the morphogen phases, and the resulting pattern (up to spatial dimension of two) can all be determined. Moreover, by incorporating spatial and temporal heterogeneities, it is shown how mixed mode patterns can be generated, allowing for arbitrarily complex patterns to be produced. The implications of these results are then discussed in the light of linking theory and experiment.

Much of Murray's pioneering work features multi-stable systems, where nonlinear dynamics can drive multiple possible outcomes. Koch et al. (2021) analyse a classical multi-stable system, mountain pine beetle populations, which can jump between endemic and outbreak states. However, this work develops a new perspective on such systems by incorporating spatial autocorrelation in dispersal dynamics for the beetles. The resulting dynamical system is then fit to detailed outbreak data, shedding light on how outbreaks can sporadically arise across the landscape.

The hallmark of Murray's work on disease dynamics, such as HIV, has been to gain insight from realistic models, tailored to the specific details that can so often affect outcomes. Britton and White (2021) show how the inclusion of multiple infection classes in a honey-bee-mite-virus system is key to our understanding of spontaneous transitions between disease states as bees and mites move from covertly to overtly infected states. These distinct infection classes then play a key role in governing disease outcomes. Nick Britton very sadly passed away in December 2020, while this paper was still in the review stage. On his request, Jane White, his long-time colleague and friend at the University of Bath, revised and finalised the manuscript in response to reviewer comments.

A unifying feature of Murray's varied research work is the use of simple models to explain complex biological phenomena. He showed that low order systems of ordinary and partial differential equations can capture the essence of processes such as embryonic development, tumour growth and disease spread. Kempes et al. (2021) applies this philosophy to the field of astrobiology. The paper uses a simple ordinary differential equation model coupled with previously established scaling relationships of the main macromolecular components of cells. This approach enables prediction of potential biosignatures of life, via variation of elemental ratios in a range of terrestrial biological environments.

Villa et al. (2021) re-examines a key aspect of the Murray-Oster mechanochemical theory of pattern formation, namely that of the role of the constitutive equation. In 
the original theory the main assumption of the modelling regarding the biomechanical properties of tissue was that it was a linear viscoelastic material governed by the socalled Kelvin-Voigt model. This linear stress-strain relationship permits an equation to be derived for the displacement of the tissue (cell-extracellular matrix system) which, in turn, is used to derive a dispersion relation, whence a prediction of pattern formation. The results of the current paper show that pattern formation is critically dependent upon the constitutive equation chosen and that fluid-like constitutive models have a higher potential for generating patterns than solid-like constitutive models.

As mentioned above, Murray's work explored the idea that, in the context of developmental pattern formation, evolution can be captured by movement, on a vastly different timescale, through parameter space. Of course, at the macroscale level, parameters are largely a model construct as they really describe processes occurring on different length and/or timescales. How to link processes across different scales is now a major change in the field of mathematical biology. Stotsky et al. (2021) use multiscale continuous time random walks and generalised master equations to go from the microscale to the macroscale in the context of transport processes in tissues. It is shown, using signalling in the Drosophila wing disc as a case study, how the framework developed can be employed to directly link experimentally observable macroscale properties, such as transport coefficients, to the detailed underlying microscale level tissue properties.

A very important weapon in the armoury of the applied mathematician is asymptotic analysis, a subject which formed the basis for Murray's first book (Murray 1974) and which also plays a role in Efendiev et al. (2021). This paper investigates high intensity focussed ultrasound (HIFU), which is being increasingly used as a promising cancer treatment, without the side effects of more standard treatments. This paper investigates the long-time dynamics of a previously proposed partial differential equation system that aims to model energy deposition in biological tissue due to HIFU. Specifically, a number of theoretical results are derived on the attractors shown to exist for this system.

Pattern formation has been a central component of Murray's work. He primarily studied patterns in embryonic development, but his ideas have subsequently been exported to a wide range of other biological contexts. Sherratt et al. (2021) considers pattern formation in intertidal mussel beds, where the patterning occurs at the scale of the whole ecosystem. The paper makes a detailed comparison between two different hypothesised mechanisms for patterning, and demonstrates important differences between the resulting patterns for both biomass distribution and resilience to disturbances.

Lui and Myerscough (2021) address one of the few research areas in which Murray has not worked, namely, heart disease. This paper proposes a model for the composition of atherosclerotic plaques, which can cause heart attacks and strokes. More specifically, it develops an ordinary differential equation model for time evolution of macrophage cells and lipids. It is shown that the model is amenable to a multiple timescale analysis and the contribution of key cell processes to plaque lipid accumulation is explored in detail.

Fowler (2021) extends the aforementioned work of Murray et al. (1986) on rabid foxes to address the issue of extinction, which is a general problem faced by con- 
tinuous models of population dynamics, whose applicability is called into question at low numbers of individuals. A number of ways to address/resolve these problems is presented in this paper, and illustrated through application to foxes approaching extinction; oscillatory dynamics with extremely small minima, explored in the context of immune dynamics and microbial growth models; and finally frogspawn, where an age-structured model is proposed and analysed.

The articles in this Special Collection illustrate the synergy between the fields of mathematics and biology (in its broadest sense) that J.D. Murray has demonstrated countless times thoroughout his amazing career: biology inspires new mathematics, while mathematics leads to new biology.

\section{References}

Anita S, Capasso V, Scacchi S (2021) Controlling the spatial spread of a Xylella epidemic. Bull Math Biol 83:22

Bentil DE, Murray JD (1993) Modelling bovine tuberculosis in badgers. J Anim Ecol 62:239-250

Britton NF, White KAJ (2021) The effect of covert and overt infections on disease dynamics in honey-bee colonies. Bull Math Biol 83:67

Burgess PK, Kulesa PM, Murray JD, Alvord EC Jr (1997) The interaction of growth rates and diffusion coefficients in a three-dimensional mathematical model of gliomas. J Neuropathol Exp Neurol 56:704713

Cassidy T, Humphris AR, Craig M, Mackey MC (2020) Characterizing chemotherapy-induced neutropenia and monocytopenia through mathematical modelling. Bull Math Biol 82:104

Cook J, Tyson R, White KAJ, Rushe R, Gottman J, Murray JD (1995) Mathematics of marital conflict: qualitative dynamic mathematical modeling of marital interaction. J Fam Psycol 9(2):110-130

Curtin L, Hawkins-Daarud A, Porter AB, van der Zee KG, Owen MR, Swanson KR (2020) A mechanistic investigation into ischemia-driven distal recurrence of glioblastoma. Bull Math Biol 82:143

Fowler AC (2021) Atto-foxes and other minutiae. Bull Math Biol 83:104

Gottman JM, Murray JD, Swanson CC, Tyson R, Swanson KR (2002) The mathematics of marriage: dynamic nonlinear models. MIT Press, Cambridge

Kempes CP, Follows MJ, Smith H, Graham H, House CH, Levin SA (2021) Generalised stoichiometry and biogeochemistry for astrobiological applications. Bull Math Biol 83:73

Koch D, Lewis MA, Lele S (2021) The signature of endemic populations in the spread of mountain pine beetle outbreaks. Bull Math Biol 83:65

Kulesa PM, Kasemeier-Kulesa JC, Morrison JA, McLennan R, McKinney MC, Bailey C (2021) Modelling cell invasion: a review of what JD Murray and the embryo can teach us. Bull Math Biol 83:26

Lane DC, Murray JD, Manoranjan VS (1987) Analysis of wave phenomena in a morphogenetic mechanochemical model and an application to post-fertilization waves on eggs. IMA J Math Appl Med Biol 4:309-331

Levy B, Correia HE, Chirove F, Ronoh M, Abebe A, Kgosimore M, Chimbola O, Machingauta MH, Lenhart S, White KAJ (2021) Modeling the effect of HIV/AIDS stigma on HIV infection dynamics in Kenya. Bull Math Biol 83:55

Lewis MA, Murray JD (1993) Modelling territoriality and wolf-deer interactions. Nature 366(6457):738740

Lui G, Myerscough MR (2021) Modelling preferential phagocytosis in atherosclerosis: delineating timescales in plaque development. Bull Math Biol 83:96

Murray JD (1974) Asymptotic analysis. Clarendon Press, Oxford (further material added in asymptotic analysis, applied mathematical sciences, vol 48, Springer-Verlag, New York, 1984, 2nd printing, 1996)

Murray JD (1981) A pre-pattern formation mechanism for animal coat markings. J Theor Biol 88:161-199

Murray JD (1988) Mammalian coat patterns: how the leopard gets its spots. Sci Am 256:80-87

Murray JD (2002) Mathematical biology: I. An introduction 2002 (3rd Edition). Interdisciplinary applied mathematics, vol 17. Springer 
Murray JD (2003) Mathematical biology: II. Spatial models and biomedical applications (3rd Edition). Interdisciplinary applied mathematics, vol 18. Springer

Murray JD (2004) Mathematical biology, 3rd edition in 2 volumes: mathematical biology: I. An introduction 2002 (2nd printing 2004) interdisciplinary applied mathematics, vol 17. Springer

Murray JD (2008) Mathematical biology: II. Spatial models and biomedical applications 2003 (2nd printing 2004, 3rd printing 2008) interdisciplinary applied mathematics, vol 18. Springer

Murray JD, Deeming DC, Ferguson MWJ (1990) Size dependent pigmentation pattern formation in embryos of alligator mississippiensis: time of initiation of pattern generation mechanism. Proc Roy Soc Lond B 239:279-293

Murray JD, Myerscough MR (1991) Pigmentation pattern formation on snakes. J Theor Biol 149:339-360

Murray JD, Oster GF, Harris AK (1983) A mechanical model for mesenchymal morphogenesis. J Math Biol 17:125-129

Murray JD, Stanley EA, Brown DL (1986) On the spatial spread of rabies among foxes. Proc Roy Soc Lond B 229:111-150

Nelson P, Perelson AS, Murray JD (2000) Delay model for the dynamics of HIV infection. Math Biosci 163:201-215

Oster GF, Murray JD, Harris AK (1983) Mechanical aspects of mesenchymal morphogenesis. J Embryol Exp Morph 78:83-125

Oster GF, Shubin N, Murray JD, Alberch P (1988) Evolution and morphogenetic rules: the shape of the vertebrate limb in ontogeny and phylogeny. Evolution 42:862-884

Seirin-Lee S (2021) The role of cytoplasmic MEX-5/6 polarity in asymmetric cell division. Bull Math Biol $83: 29$

Sherratt JA, Ling Q-X, van de Koppel J (2021) A comparison of the "reduced losses" and "increased production" models for mussel bed dynamics. Bull Math Biol 83:99

Sherratt JA, Martin P, Murray JD, Lewis J (1992) Mathematical models of wound healing in embryonic and adult epidermis. IMA J Math Appl Med Biol 9:177-196

Sherratt JA, Murray JD (1990) Models of epidermal wound healing. Proc Roy Soc Lond B 241:29-36

Sherratt JA, Murray JD (1992) Epidermal wound healing: the clinical implications of a simple mathematical model. Cell Transpl 1:365-371

Sneyd J, Atri A, Ferguson MWJ, Lewis MA, Seward W, Murray JD (1993) A model for the spatial patterning of teeth primordia in the alligator: initiation of the dental determinant. J Theor Biol 165:633-658

Sneyd J, Vera-Sigüenza E, Rugis J, Pages N, Yule DI (2021) Calcium dynamics and water transport in salivary acinar cells. Bull Math Biol 83:31

Stotsky JA, Gou J, Othmer HG (2021) A random walk approach to transport in tissues and complex media: from microscale descriptions to macroscale models. Bull Math Biol 83:92

Swanson KR, Alvord EC Jr, Murray JD (2000) A quantitative model for differential motility of gliomas in grey and white matter. Cell Prolif 33:317-329

Swanson KR, Alvord EC Jr, Murray JD (2002) Virtual brain tumors (gliomas) enhance the reality of medical imaging and highlight inadequacies of current therapy. Br J Cancer 86:14-18

Swanson KR, Bridge C, Murray JD, Alvord EC Jr (2003) Virtual and real brain tumors: using mathematical modeling to quantify glioma growth and invasion. J Neurol Sci 216(1):1-10

Tracqui P, Cruywagen GC, Woodward DE, Bartoo GT, Murray JD, Alvord EC Jr (1995a) A mathematical model of glioma growth: the effect of chemotherapy on spatial-temporal growth. Cell Prolif 28:17-31

Tracqui P, Woodward DE, Cruywagen GC, Cook J, Murray JD (1995b) A mechanical model for fibroblastdriven wound healing. J Biol Syst 3:1075-1085

Turing AM (1952) The chemical basis of morphogenesis. Philos Trans Roy Soc B 237:37-72

Villa C, Chaplain MAJ, Gerisch A, Lorenzi T (2021) Mechanical models of pattern and form in biological tissues: the role of stress-strain constitutive equations. Bull Math Biol 83:8

White KAJ, Lewis MA, Murray JD (1996a) A model for wolf-pack territory formation and maintenance. J Theor Biol 178:29-43

White KAJ, Murray JD, Lewis MA (1996b) Wolf-deer interactions: a mathematical model. Proc R Soc Lond B 263:299-305

Woodward DE, Cook J, Tracqui P, Cruywagen GC, Murray JD, Alvord EC Jr (1996) A mathematical model of glioma growth: the effect of extent of surgical resection. Cell Prolif 29:269-288

Woolley TE, Krause AL, Gaffney EA (2021) Bespoke Turing systems. Bull Math Biol 83:41 
Publisher's Note Springer Nature remains neutral with regard to jurisdictional claims in published maps and institutional affiliations. 